\title{
Error Analysis of Boundary Condition Approximations in the Modeling of Coaxially-Gated Carbon Nanotube Field-Effect Transistors
}

\author{
Dylan L. McGuire ${ }^{1}$ and David L. Pulfrey*1 \\ 12356 Main Mall, Vancouver, BC Canada V6T 1 Z4
}

Received 15 November 2003, revised 30 November 2003, accepted 2 December 2003

Published online 3 December 2003

\begin{abstract}
Key words carbon nanotubes, modeling, boundary condition, error analysis. PACS 04A25

In the modeling of carbon nanotube field-effect transistors, non-physical boundary conditions are often employed at the borders of the simulation space. This paper investigates the consequences of imposing these boundary conditions on common geometries, and proposes solutions which reduce the error without compromising simulation efficiency.
\end{abstract}

Copyright line will be provided by the publisher

\section{Introduction}

The modeling of carbon nanotube field-effect transistors (CNFETs) is an active area of research, one of the present goals of which is to evaluate the performance limits of devices as regards operation under conditions of either DC [1,2], or AC [3]. The majority of modeling work thus far has concentrated on coaxial transistors with wrap-around gates, as such a structure offers the ultimate gate control over the nanotube surface potential [4]. Fabrication of such structures is difficult, but is being vigorously pursued [5, 6]. In the numerical modeling of semiconductor devices it is customary to use a null Neumann boundary condition, in which the normal component of the electric field set to zero, to terminate the open boundaries of the model space. In nanoscale devices, this practice is widely followed $[3,7,8]$, although strict null Neumann boundaries are not typically found $a$ priori. In this study, an asymptotic analysis examining the electric field near the carbon nanotube $(\mathrm{CN})$-contact interface reveals that a Cartesian formulation of Poisson's equation is applicable in the local region and demonstrates that a null Neumann boundary is not physically correct.

When the symmetry of the device allows a reduction in dimension to 2-D, a conformal transformation yields an exact solution for the potential in all regions [9]. We use this method as a benchmark, solving the cylindrical Poisson equation in all space, and compare against results obtained from imposing a null Neumann condition at open boundaries in Schottky-barrier (SB) CNFETs. We consider the error introduced in the DC ON-current, extending the earlier work of Ref. [9], in which only the equilibrium case was considered. We continue to evaluate a coaxial structure as a benchmark device in terms of scale and performance, although the device dimensions are based on a state-of-the-art planar device [10], with short CN length, high-permittivity gate dielectric, and thin source/drain contacts. We consider the effect of several geometry modifications on the accuracy of the results, including reductions to the radius of the source/drain contacts, changes to the gate dielectric thickness and material, and changes to the spacing between the source/drain electrodes and the edge of the gate electrode.

* Corresponding author: e-mail: pulfrey@ece.ubc.ca, Phone: +01 604822 3876, Fax: +01 6048225949 


\section{Modeling Procedures}

Fig. 1 illustrates the coaxial structure simulated in this work: the 2-D Poisson equation is solved using a standard finite-element software package ${ }^{1}$, and the effective-mass Schrödinger equation is solved selfconsistently in 1-D to compute the charge distribution [11]. The current is computed using the Landauer equation [12]. In one instance, null Neumann boundary conditions are used at the open boundaries (see Fig. 1 (b), dashed line); in the benchmark case, the conformal transformation method described in Ref. [9] is used. Solving the conformally mapped problem numerically, error sources are restricted to the convergence tolerance and the element size.

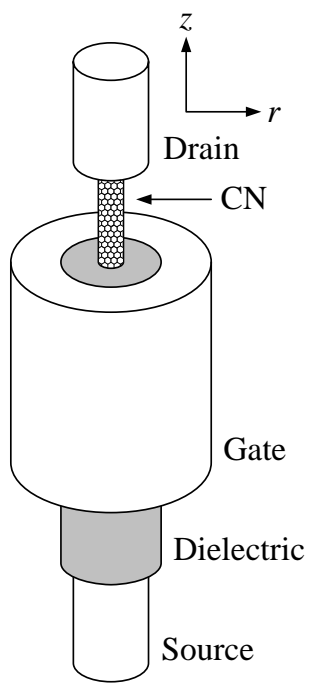

(a)

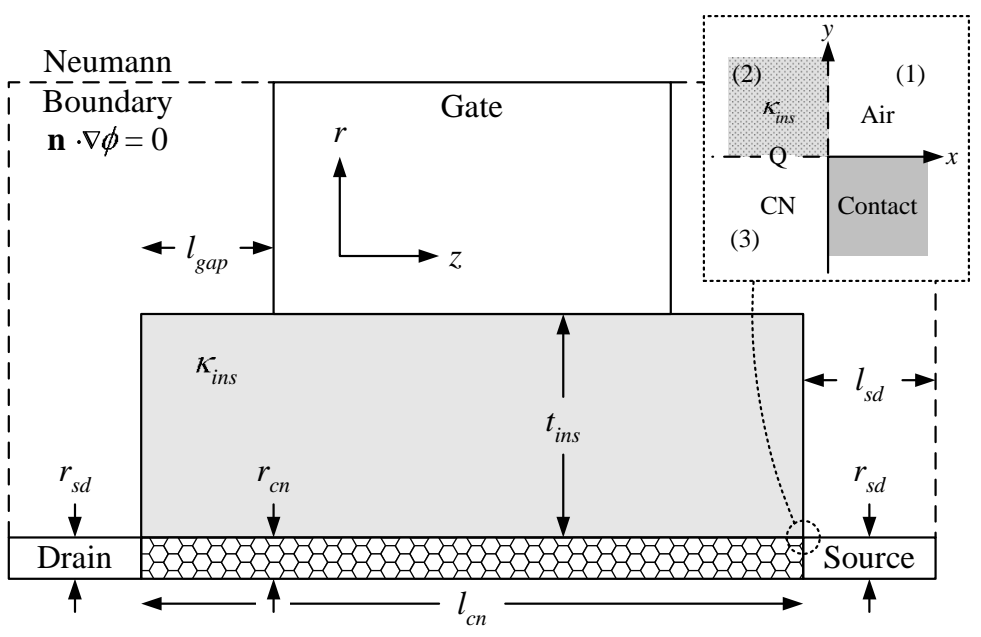

(b)

Fig. 1 Geometry overview showing (a) the cylindrical device and (b) the radial cross-section including key device parameters. The dashed line indicates the location of the null Neumann condition used to close the open boundaries. A non-zero $l_{s d}$ is illustrated although typically, $l_{s d}=0$. The inset shows the important region at the contact/nanotube interface and the regional numbering scheme used in the text.

As discussed in the following section, we observed the largest error when using "needle" contacts, i.e. $r_{s d}=r_{c n}$. Therefore, we performed an asymptotic analysis of the potential at the CN-contact interface near the CN surface to understand how this error may be reduced. This analysis follows the method presented in Ref. [13, pp. 75-79], and extends it by including both a dielectric and a surface charge. The surface charge $Q$ is assumed to be constant in the region of interest.

We begin by establishing that sufficiently close to the contact, where $r / r_{c n} \approx 1$, a Cartesian formulation of Poisson's equation is valid. The situation is illustrated in the inset of Fig. 1 (b). Defining local coordinates

$$
x=\frac{z-z_{0}}{\delta} \quad \text { and } \quad y=\frac{r-r_{c n}}{\delta},
$$

where $\delta \ll r_{c n}$ and $z_{0}$ is the location of the $\mathrm{CN}$-contact interface, Poisson's equation (for potential $\phi(z, r)$ ) in cylindrical coordinates becomes

$$
\frac{\partial^{2} \phi}{\partial x^{2}}+\frac{\partial^{2} \phi}{\partial y^{2}}+\frac{\delta}{\delta y+r_{c n}} \frac{\partial \phi}{\partial y}=\delta^{2} \frac{Q}{\epsilon} .
$$

\footnotetext{
${ }^{1}$ FEMLAB, see $h t t p: / / w w w . c o m s o l . c o m$
} 
As $r / r_{c n} \rightarrow 1, \delta y \ll r_{c n}$ and the coefficient of the first partial derivative is $\mathrm{O}(\delta)$. For a small $\delta$, we conclude that the cylindrical Poisson equation can be locally approximated by the Cartesian Laplacian.

Based on this conclusion, we proceed by transforming the Cartesian Laplacian into polar coordinates and solving for the potential $\phi(\sigma, \theta)$. Separation of variables is used to break the problem into a radial equation and an angular equation with a well-known series solution [13], which can be written in terms of one arbitrary constant $K$, which is related to the dielectric constant $\kappa_{\text {ins }}$, boundary layer charge $Q$ (see inset, Fig. $1 \mathrm{~b}$ ) and boundary conditions as $\sigma \rightarrow \infty$. In this analysis, no attempt is made to join the inner and outer regions, leaving the boundary conditions where $\sigma \rightarrow \infty$ undefined. Therefore, $K$ may be treated as a geometry-dependent parameter chosen to account for the influence of structures in the outer region, e.g. the gate, on the electric field. Assuming that $\sigma \ll 1$, and that $K_{j}$ increases monotonically with $j$, the series index, we approximate the general solution by the first solution in the series, yielding

$$
\phi(\sigma, \theta) \simeq a_{0}+a_{i} f_{i}(K) \sigma^{K}\left[g_{i}(K) \cos (K \theta)+\sin (K \theta)\right],
$$

where $i$ is a domain index. $f_{i}(K)$ and $g_{i}(K)$ represent the replacement of other constants in terms of $K$, and may depend on $\kappa_{i n s}$ and $Q$. This provides a means for including the iteratively computed charge in the approximation to the electric field at a selected boundary.

This analysis provides two methods for reducing error introduced by the null Neumann boundary. Taking the derivative of Eq. 3 normal to the Neumann boundary at the location of the CN-contact interface,

$$
\begin{aligned}
\left.\frac{\partial \phi}{\partial x}\right|_{\mathrm{i}}= & a_{i} y K f_{i}(K)\left[g_{i}(K) \sin \left(K \tan ^{-1} \frac{y}{x}\right)-\cos \left(K \tan ^{-1} \frac{y}{x}\right)\right]\left(x^{2}+y^{2}\right)^{K / 2-1} \\
& +2 x f_{i}(K)\left[g_{i}(K) \cos \left(K \tan ^{-1} \frac{y}{x}\right)+\sin \left(K \tan ^{-1} \frac{y}{x}\right)\right]\left(x^{2}+y^{2}\right)^{K / 2}
\end{aligned}
$$

At the CN-contact interface of a needle-contacted device, $x=0$, and Eq. 4 implies that the normal gradient of the potential will decay proportional to $y^{K-1}$, where $K$ is chosen based on the geometry and it is implict that $K<1$. Therefore, replacing the null Neumann boundary with a normal gradient that varies as $\left(r-r_{c n}\right)^{K-1}$ will better approximate the physical boundary conditions, locally.

Alternately, we observe that by extending the simulation space to include a portion of the contact $\left(l_{s d}>\right.$ 0 ), the error can be reduced. Near the surface of the $\mathrm{CN}$ and, equivalently, the surface of the contact in region I, $y \ll 1$, and $\theta=\tan ^{-1}(x / y) \approx 0$. As $x$ is increased, such that $x \gg y$, Eq. 4 can be simplified to

$$
\left.\frac{\partial \phi}{\partial x}\right|_{\mathrm{I}} \approx-a_{i} K f_{1}(K) x^{K-1}
$$

in the valid range of the local approximation. With $K<1$, Eq. 5 suggests that as $x$ is increased near the surface of the contact, the gradient of the potential in the direction normal to the boundary approaches zero.

\section{Results and Discussion}

Results are presented for the five SB-CNFETs listed in Table 1. Note that all devices except for device 1 use a $(10,0) \mathrm{CN}$. These latter devices have larger bandgaps, resulting in FETs with significantly improved ON/OFF-current ratios [1]. The errors in the potential and drain current, evaluated with respect to the results obtained using the conformal transformation are summarized in Table 2. The maximum error in the potential was computed by taking the infinity norm of the relative error at all positions on the $\mathrm{CN}$ surface. In all cases, the greatest deviation between potentials occurred close to the source. The valence band diagrams in the vicinity of the source for each of devices 1-4 are shown in Fig. 2.

For devices 1 (circle) and 2 (square), there is no discernible difference in potential, however the relative ON-current error in device 2 is higher because tunneling dominates in this positive-barrier device, and is exponentially dependent on the shape of the barrier. Devices 3 (triangle) and 4 (diamond) illustrate the 
Table 1 Device Properties. In all cases $l_{c n}=50 \mathrm{~nm}$, and the $\mathrm{CN}$ work function is $4.73 \mathrm{eV}$ [14]. Devices 1 and 2 have Pd end contacts with barrier heights obtained from Ref. [15], while devices 3-5 have $(9,0)$ metallic $\mathrm{CN}$ contacts. Geometry dimensions are referenced to Fig. 1 (b), $E_{g}$ is the CN bandgap, and $\Phi_{B}$ is the SB height for holes.

\begin{tabular}{c|cccccccc}
\hline Device & $r_{c n}(\mathrm{~nm})$ & $r_{s d}(\mathrm{~nm})$ & $t_{i n s}(\mathrm{~nm})$ & $l_{g a p}(\mathrm{~nm})$ & $\kappa_{i n s}$ & Chirality & $E_{g}(\mathrm{eV})$ & $\Phi_{B}(\mathrm{eV})$ \\
\hline $1[10]$ & 0.85 & $6.15^{*}$ & 8.00 & 8.00 & 16 & $(22,0)$ & 0.46 & -0.04 \\
2 & 0.39 & $5.69 * *$ & 8.00 & 8.00 & 16 & $(10,0)$ & 0.98 & +0.30 \\
3 & 0.39 & 0.39 & 8.00 & 8.00 & 16 & $(10,0)$ & 0.98 & +0.49 \\
4 & 0.39 & 0.39 & 2.00 & 12.0 & 16 & $(10,0)$ & 0.98 & +0.49 \\
5 & 0.39 & 0.39 & 8.00 & 8.00 & 25 & $(10,0)$ & 0.98 & +0.49 \\
\hline
\end{tabular}

significant error that arises from non-physical boundaries near needle contacts. In the case of the aspect ratio of the gate and source/drain contact gap to the dielectric thickness, comparing devices 3 and 4, we note that by reducing $l_{\text {gap }}$ or increasing $t_{\text {ins }}$, the electric field between the gate and the source/drain contact is oriented in a more radial direction, reducing the component normal to boundary. This makes the null Neumann boundary a better approximation, reducing error. From the flux continuity condition, it is clear that greater refraction will exist when a high- $\kappa$ gate dielectric is present, reducing the normal component of the potential gradient. In device 5, a $56 \%$ increase in $\kappa$ with respect to device 3 led to a $44 \%$ decrease in relative $\mathrm{ON}$-current error.

Table 2 Error in potential and drain current for bias conditions of $V_{G S}=V_{D S}=-0.5 \mathrm{~V}$

\begin{tabular}{c|cc}
\hline Device & Max. V Error (\%) & $\mathbf{I}_{\mathbf{D}}$ Error (\%) \\
\hline 1 & 0.24 & 0.62 \\
2 & 0.36 & 4.13 \\
3 & 26.6 & 62.7 \\
4 & 42.2 & 435 \\
5 & 21.2 & 43.4 \\
\hline
\end{tabular}

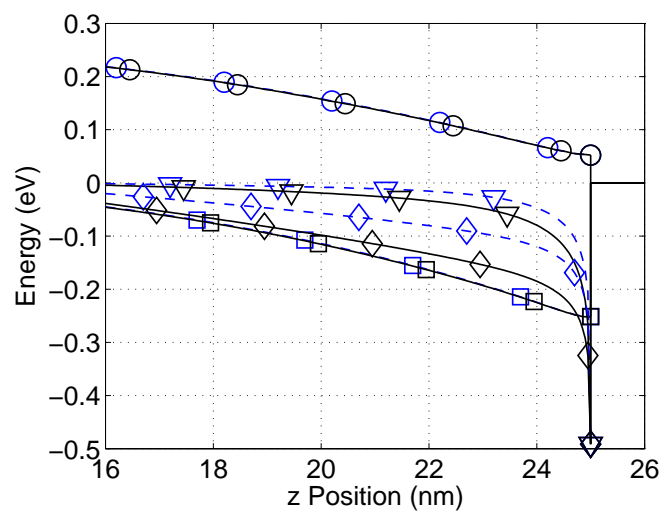

Fig. 2 Valence band diagrams for devices 1 to 4 near the source contact, referenced to the Fermi level. Conformal mapped case: solid line. Null Neumann bounded: $l_{s d}=0 \mathrm{~nm}$, dashed line. Device 1: $\circ$, device $2: \square$, device 3: $\nabla$, device 4: $\diamond$

To illustrate how the large error associated with needle contacts can be reduced, we refer to the asymptotic analysis. With $K<1$, Eq. 5 suggests that as $x$ is increased near the surface of the contact, the gradient of the potential in the direction normal to the boundary will approach zero. This coincides with the intuitive picture that a flat plate behaves like an infinite plane when observed at a position far from the corners: the equipotential lines are parallel to the plate's surface. By including a portion of the contact in the simulation, and imposing the null Neumann boundary condition further from the $\mathrm{CN}$-contact interface, the error from the non-phyisical boundary will be reduced. To support this hypothesis, simulations were performed for device 4 (the one with the largest error), with $l_{s d}$ extended beyond the value $0 \mathrm{~nm}$ used in the previous simulations. Results are shown in Fig. 3 (a). In Fig. 3 (b), we demonstrate the validity of the asymptotic analysis in the prediction of the local electric field. Extending the simulation space, we note that 
a limit in the reduction of error is reached at $\approx 2.5 \%$. Based on the predictions of the asymptotic analysis and the simulation results, we conclude that for contacts with the same radius as the $\mathrm{CN}$, the boundary should be extended to include $l_{s d} \geq 10 r_{c n}$ of the contact to reduce the relative ON-current error below $10 \%$ or $l_{s d} \geq 20 r_{c n}$ to ensure that the error remains below $5 \%$. These metrics are arrived at for the worst case $\mathrm{CN}$-contact interface geometry and will apply conservatively for contacts with $r_{s d}>r_{c n}$.

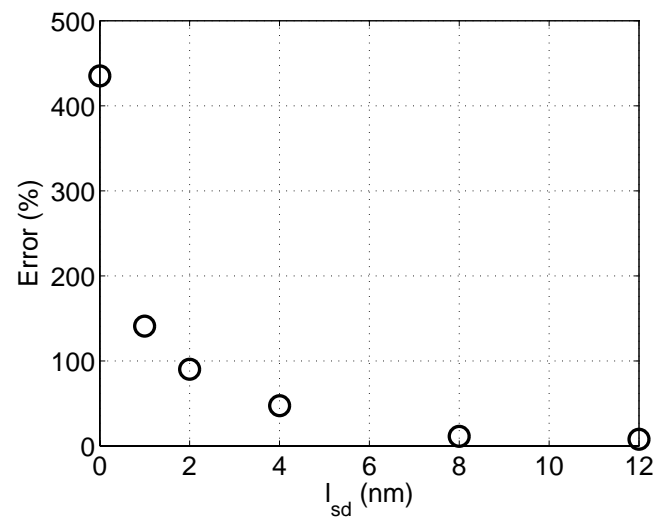

(a)



(b)

Fig. 3 Asymptotic analysis results for device 4. (a) Simulated relative ON-current error as $l_{s d}$ is increased, which decays as an exponential $\propto l_{s d}^{K-1}, K=0.15$ (see Eq. 5). It is assumed that as the electric field at the boundary approaches zero, the error will decrease proportionally. (b) Comparison of $\frac{d \phi}{d z}$ (circles) as computed at the CN-contact interface (needle contacts) to the asymptotic approximation (solid) with $K=0.15$. Away from the contact, the local approximation is less valid. In the far field, we find it convenient to use $\frac{d \phi}{d z} \propto r^{-1}$ as in an infinite rod to describe the electric field.

\section{Conclusions}

From this error analysis of methods used to compute the potential in co-axial SB-CNFETs, we conclude that caution must be exercised when employing non-physical boundaries in cases where narrow-radius contacts are present. The error can be significantly reduced, without unduly compomising the simulation efficiency, by extending the simulation space to include a length of contact.

\section{References}

[1] J. Guo, S. Datta, and M. Lundstrom, IEEE Trans. Electron Devices 51(2), 172 (2004).

[2] L. C. Castro, D. L. John, and D. L. Pulfrey, Smart Materials and Systems (2005). Accepted, July 2005. [Online.] Available: http://nano.ece.ubc.ca/pub/publications.htm.

[3] L. C. Castro, D. L. John, D. L. Pulfrey, M. Pourfath, A. Gehring, and H. Kosina, IEEE Trans. Nanotechnol. (2005). Accepted, July 2005. [Online.] Available: http://nano.ece.ubc.ca/pub/publications.htm.

[4] C. P. Auth and J. D. Plummer, IEEE Electron Device Lett. 18(2), 74 (1997).

[5] W. Hoenlein, F. Kreupl, G. Duesberg, M. Liebau, R. Seidel, and E. Unger, IEEE Trans. Components Packaging Technology 27, 629 (2004).

[6] W. Choi, E. Bae, D. Kang, S. Chae, B.-H. Cheong, J.-H. Ko, E. Lee, and W. Park, Nanotechnology 15, S512 (2004).

[7] J. Guo, J. Wang, E. Polizzi, S. Datta, and M. Lundstrom, IEEE Trans. Nanotechnol. 2(4), 329 (2003).

[8] E. Ungersboeck, M. Pourfath, H. Kosina, A. Gehring, B.-H. Cheong, W.-J. Park, and S. Selberherr, IEEE Trans. Nanotechnol. (2004). Accepted, October 2004.

[9] J. P. Clifford, D. L. John, L. C. Castro, and D. L. Pulfrey, IEEE Trans. Nanotechnol. 3(2), 281 (2004).

[10] A. Javey, J. Guo, D. B. Farmer, Q. Wang, E. Yenilmez, R. G. Gordon, M. Lundstrom, and H. Dai, Nano Lett. 4(7), 1319 (2004). 
[11] D. L. John, L. C. Castro, P. J. S. Pereira, and D. L. Pulfrey, in: Proc. NSTI Nanotech (2004), vol. 3, pp. $65-68$.

[12] D. L. John, L. C. Castro, J. P. Clifford, and D. L. Pulfrey, IEEE Trans. Nanotechnol. 2(3), 175 (2003).

[13] J. D. Jackson, Classical Electrodynamics (John Wiley and Sons, Toronto, 1999), 3rd ed.

[14] J. Zhao, J. Han, and J.-P. Lu, Phys. Rev. B 65, 193401 (2002).

[15] Z. Chen, J. Appenzeller, J. Knoch, Y. Lin, and P. Avouris, Nano Lett. 5(7), 1497 (2005). 